Check for updates

Cite this: Nanoscale Adv., 2019, 1, 4422

\title{
A multiwavelength emission detector for analytical ultracentrifugation $\uparrow$
}

\author{
Simon E. Wawra, ${ }^{a}$ Georgy Onishchukov, ${ }^{a}$ Maria Maranska, ${ }^{b}$ Siegfried Eigler, ${ }^{b}$ \\ Johannes Walter ${ }^{\mathrm{ac}}$ and Wolfgang Peukert (D)*ac
}

In this study, a new detector for multiwavelength emission analytical ultracentrifugation (MWE-AUC) is presented, which allows measuring size- or composition-dependent fluorescence properties of nanoparticle ensembles. Validation of the new setup is carried out via comparison to a benchtop photoluminescence spectrometer and the established extinction-based multiwavelength analytical ultracentrifuge (MWL-AUC). The results on fluorescent proteins and silica particles demonstrate that the new device not only correctly reproduces sedimentation and diffusion coefficients of the particles but provides also meaningful fluorescence spectra. As an application example for a sample exhibiting a broad particle size distribution, spectra and size of graphene oxide nanoplatelets are extracted simultaneously. Narrowly distributed CdSe/ZnS quantum dots showing size- and structure-dependent shifts of their fluorescence spectra are analyzed as well. The combination of MWE- and MWL-AUC provides a comprehensive framework for the optical characterization for nanoparticles and macromolecules in terms of their extinction and emission properties.

Received 7th August 2019

Accepted 3rd October 2019

DOI: $10.1039 / c 9 n a 00487 d$

rsc.li/nanoscale-advances dependent refraction index via an interferometer was put into place ${ }^{8}$ as well as the detection of its radial gradient via Schlieren optics. ${ }^{9}$ Additionally, integral fluorescence intensities of particle and protein ensembles were used for analysis in AUC. ${ }^{\mathbf{1 0 - 1 2}}$ Combining spectral information at several wavelengths ${ }^{13}$ led to the development of multiwavelength extinction AUC (MWL-AUC) ${ }^{\mathbf{1 4}}$ extending AUC analysis towards complex and often coupled size-dependent hydrodynamic, thermodynamic and optical particle properties opening up vast new possibilities, including e.g. the identification of different species ${ }^{4,5}$ or the determination of two-dimensional size distributions of anisotropic plasmonic particles. ${ }^{3}$ Even wide property distributions can now be analyzed by gravitational sweep experiments at constant radial position..$^{\mathbf{4 1 5}, \mathbf{1 6}}$ So far unexplored further potential in terms of accessible data lies in size-dependent fluorescence light detection in dependence of the excitation wavelength. At the moment, any spectral information in the formerly commercially sold fluorescence AUC cannot be acquired due to the usage of a photomultiplier with a longpass or broadband bandpass spectral filter. Without the spectral information at hand, species labelled with different fluorescent dyes cannot be easily discriminated. Therefore, multi-component detection of protein complexes can currently only be achieved by using photo-switchable proteins. ${ }^{17}$ Nevertheless, the method is very popular as it allows to study protein interactions at nanomolar concentrations. ${ }^{18}$ Apart from the patented concept ${ }^{19}$ itself, multiwavelength emission analytical ultracentrifuge (MWE-AUC) was not

demonstrated yet. In this contribution, we present a novel

\footnotetext{
${ }^{a}$ Institute of Particle Technology (LFG), Friedrich-Alexander-Universität Erlangen-Nürnberg (FAU), Cauerstrasse 4, 91058 Erlangen, Germany. E-mail: wolfgang.peukert@fau.de

${ }^{b}$ Institute for Chemistry and Biochemistry, Freie Universität Berlin, Takustraße 3, 14195, Berlin, Germany

${ }^{C}$ Interdisciplinary Center for Functional Particle Systems (FPS), Friedrich-AlexanderUniversität Erlangen-Nürnberg (FAU), Haberstrasse 9a, 91058 Erlangen, Germany

$\dagger$ Electronic supplementary information (ESI) available. See DOI: 10.1039/c9na00487d
} 
setup for multiwavelength fluorescence detection in AUC and highlight a few of its capabilities using fluorescent dyelabelled proteins and silica particles, graphene oxide as well as CdSe/ZnS quantum dots.

\section{Materials and methods}

\section{Photoluminescence spectrometer}

Measurements of the emission spectra of samples at ambient conditions were performed with a FluoroLog-3 photoluminescence spectrometer from Horiba.

\section{UV/Vis spectrometer}

Extinction data was acquired with a Specord 210 Plus from Analytik Jena.

\section{MWE-AUC}

An Optima XL-80K ultracentrifuge from Beckman Coulter was equipped with a custom-made fluorescence setup. The excitation wavelength was $518 \mathrm{~nm}$. Details on the used parts can be found in the ESI. $\dagger$

\section{MWL-AUC}

For the MWL-AUC experiments, a user-modified preparative ultracentrifuge from Beckman Coulter (Optima L-90K) equipped with a multiwavelength extinction detector was used. ${ }^{22}$ For all experiments two-sector titanium centerpieces with an optical path length of $12 \mathrm{~mm}$ from Nanolytics were used. Temperature was kept constant at $20{ }^{\circ} \mathrm{C}$.

\section{Graphene oxide}

Graphene oxide was synthesized following the modified Hummers' method. ${ }^{20,21}$ Graphite was dispersed in concentrated sulfuric acid and potassium permanganate was slowly added under continuous stirring at temperatures below $10{ }^{\circ} \mathrm{C}$. Further steps included workup with diluted sulfuric acid, followed by addition of water and diluted hydrogen peroxide. The assynthesized graphene oxide was washed with water and delaminated using tip sonication. The dispersion was diluted to a concentration of $0.45 \mathrm{~g} \mathrm{~L}^{-1}$ prior to ultrasound processing with a UP-200S tip sonotrode from Hielscher Ultrasonics for 20 minutes (50\% and duty cycle 0.5$)$ at $10{ }^{\circ} \mathrm{C}$. After processing, the sample was further diluted $1: 1$ with Millipore water. Prior to the measurement, the sample was processed using bath sonication for 10 minutes. The sample was measured in a constant radius experiment using a constant rotor speed of $10000 \mathrm{rpm}$ and a radial position of $6.8 \mathrm{~cm}$.

\section{Silica particles}

Fluorescent silica particles (50 nm sicastar ${ }^{\circledR}$-redF with plain surface) from Micromod Partikeltechnologie were used for the experiments. Samples were diluted with Millipore water to $1 \mathrm{mg}$ $\mathrm{mL}^{-1}$ (MWE-AUC) and $5 \mathrm{mg} \mathrm{mL}^{-1}$ (MWL-AUC). Prior to any measurement, samples were processed using bath sonication for 10 minutes. Samples were measured in a constant radius experiment using a constant rotor speed of $8000 \mathrm{rpm}$ and a radial position of $6.8 \mathrm{~cm}$.

\section{Fluorescent protein}

A fluorescently labelled protein (Albumin-fluorescein isothiocyanate conjugate) from Sigma Aldrich was used for the sedimentation velocity experiments $\left(40000 \mathrm{rpm}, 20{ }^{\circ} \mathrm{C}\right)$. $0.06 \mathrm{wt} \%$ of protein was dissolved without any purification in a Tris/NaCl buffer (12 and $15 \mathrm{mM}$, respectively).

\section{Quantum dots}

CdSe/ZnS core-shell quantum dots stabilized with carboxylic acid in water $\left(\lambda_{\text {em }}=540 \mathrm{~nm}, 1 \mathrm{mg} \mathrm{mL}^{-1}\right)$ were purchased from Sigma Aldrich and studied after 10 minutes of bath sonication. Samples were measured in a sedimentation velocity experiment using a rotor speed of $35000 \mathrm{rpm}$ at $20{ }^{\circ} \mathrm{C}$.

\section{SEDFIT}

SEDFIT $^{23}$ version 16.1 was used to determine the continuous $\mathrm{c}(\mathrm{s})$ distributions. For the data evaluation, a resolution of at least 100 data points between 0.1 and $10 \mathrm{~S}$ for the protein and 200 data points between 1 and $30 \mathrm{~S}$ for the quantum dots were used. Radial- (RI) and time-invariant (TI) noise as well as the meniscus were fitted. For the fluorescence data, the signal was normalized to make it adjustable in SEDFIT using the fluorescence tools. ${ }^{18}$ Radial magnification gradient, temporal linear magnification drift, exponential photophysical intensity change, radial convolution from detection cone with excitation shadow and nonlinear detection were fitted in the first step together with RI and TI noise and the meniscus position. Then the fluorescence parameters were kept constant while RI and TI noise and the meniscus position were fitted again. SEDFIT standard parameters $\left(\bar{v}=0.73 \mathrm{~cm} \mathrm{~g}^{-1}, \rho_{\mathrm{S}}=1.0 \mathrm{~g} \mathrm{~cm}^{-1}, \eta=\right.$ 0.01002 poise) were used for the data evaluation, as the partial specific volume of the protein with attached dye is unknown. The confidence level of the regularization (max entropy for the protein and $2^{\text {nd }}$ derivative for the quantum dots) was set to 0.95 .

\section{Constant radius experiments and data evaluation via HDR- MULTIFIT}

Constant radius experiments, which are derived from gravitational sweep experiments, 15,16 allow the analysis of broad particle size distributions. This is achieved by constantly increasing the rotor speed, while evaluating the temporal evolution of the particle concentration at a constant radial position. In order to increase the signal-to-noise ratio of the detected spectra, several light flashes of low intensity can be accumulated on the detector's CCD chip prior to readout.,24 This, however, restricts the experiment to constant rotor speeds, therefore throughout this manuscript it is called constant radius experiment. This combination of AUC techniques is especially useful for the accurate determination of optical spectra and sedimentation coefficient distributions with significant tailing for larger values. It was used for the evaluation of silica and graphene oxide particles. 
Data was evaluated in HDR-MULTIFIT (available from J. Walter) using a direct boundary model $^{25}$ with 150 grid points and a regularization parameter of 0.95 , which provides nonnormalized cumulative sedimentation coefficient distributions for every evaluated emission wavelength.

\section{Results and discussion}

\section{AUC theory}

The theoretical basics of AUC can be found in the literature. ${ }^{26-28}$ Briefly, the radial and temporal evolution of the particle concentration $c$ is given by the Lamm equation ${ }^{29}$ with the radius $r$, the time $t$, the angular velocity $\omega$ and the sedimentation and diffusion coefficients $s$ and $D$ :

$$
\frac{\partial c}{\partial t}=D\left[\frac{\partial^{2} c}{\partial r^{2}}+\frac{1}{r} \frac{\partial c}{\partial r}\right]-s \omega^{2}\left[\left(r \frac{\partial c}{\partial r}+2 c\right)\right]
$$

The sedimentation coefficient $s$ is defined as the sedimentation velocity $u$ normalized to the applied centrifugal field and can be expressed in terms of particle mass $m$, partial specific volume of the particle $\bar{v}$, friction factor $f$ and solvent density $\rho_{\mathrm{S}}$ :

$$
s=\frac{u}{\omega^{2} r}=\frac{m\left(1-\bar{v} \rho_{\mathrm{S}}\right)}{f}
$$

In general, the sedimentation coefficient $s$ depends on the size and the shape of the particles under consideration as well as on their density. For spherical particles eqn (2) can be simplified, as the hydrodynamic diameter $x_{\mathrm{H}}$ within the friction factor $f=$ $3 \pi \eta x_{\mathrm{H}}$ matches the geometric particle diameter $d$. Therefore, the latter can be calculated from the measured sedimentation coefficient with the solvent viscosity $\eta$ :

$$
d=\sqrt{\frac{18 \eta s}{\frac{1}{\bar{v}}-\rho_{\mathrm{S}}}}
$$

The frictional properties also influence the diffusion coefficient $D$ :

$$
D=\frac{k_{\mathrm{B}} T}{f}
$$

The diffusion coefficient thus only depends on the friction factor $f$, which is determined by the size and shape of the particle, as well as on the thermal energy represented by the Boltzmann constant $k_{\mathrm{B}}$ and the temperature $T$. As both the sedimentation and the diffusion coefficient depend on the friction factor $f$, the combined knowledge of $s$ and $D$ can be used to derive the molar mass of the particle via the Svedberg equation. ${ }^{30}$ In terms of data analysis, it is convenient to describe the diffusion coefficient $D$ as a function of the sedimentation coefficient $s$ and the frictional ratio $f / f_{0}$, which is the ratio of the hydrodynamic to the volume equivalent diameter. The two equivalent diameters describe the size of a spherical particle having the same properties (volume or friction, respectively) as the analyzed anisotropic particle. Within the $\mathrm{c}(\mathrm{s})$ analysis method in SEDFIT, ${ }^{23}$ it is possible to vary $f / f_{0}$ or $D$, which can be used interchangeably for a single species:

$$
D(s)=k_{\mathrm{B}} T\left[2\left(\frac{1}{\bar{\nu}}-\rho\right)\right]^{\frac{1}{2}}\left[18 s^{\frac{1}{2}} \pi\left(\frac{f}{f_{0}} \eta\right)^{\frac{3}{2}}\right]^{-1}
$$

\section{Data acquisition and evaluation}

In order to determine diffusion coefficient (eqn (5)), sedimentation coefficient (eqn (2)) or particle size (eqn (3)) distributions, the measured sedimentation boundaries need to be analyzed using numerical solutions to the Lamm equation (eqn (1)). The integral sedimentation boundary results from a superposition of the sedimentation boundaries of individual species. The concentration of each fraction must be correctly represented within the distribution. Although concentration is not directly observable for MWE- and MWL-AUC, it can be calculated from the radial and temporal evolution of the acquired optical signal. Data from MWL-AUC, for instance, can be analyzed using the extinction coefficient, which depends on the complex refractive index as well as on particle size and shape. However, even without this coefficient, the individual fractions can be weighted according to their optical signal, which allows determining extinction or emission weighted sedimentation coefficient distributions for all available wavelengths. Vice versa, emission or extinction spectra can be extracted for individual species as can be seen in Fig. 1.

Additionally to varying weighting due to different optical signals, the concentration sensitivities of MWL- and MWE-AUC are different. MWL-AUC has the advantage of a free choice of wavelength in a broad spectral range, which allows obtaining data of good quality in the majority of studies. This is more complicated in the case of MWE-AUC because the optical response for MWE-AUC depends heavily on the optical properties of the sample in proximity of the excitation wavelength (see Fig. 1). Especially, the inner filter effect can lead to a nonlinear relation between emission signal and concentration. This happens because the sample extinction decreases the excitation intensity on the way to the light focus in the sample as well as the intensity of the emitted light on the way to the detector. The inner filter effect can be avoided by using low concentrations. However, in order to obtain sufficient signal from weakly emitting samples, strong excitation may be required, which can lead to photodegradation and an distortion of the sedimentation coefficient distribution.

\section{Optics and movement of excitation source}

The MWE-AUC design, as shown in Fig. 2, is based partly on concepts used for MWL-AUC ${ }^{31}$ and the established confocal fluorescence detector. ${ }^{12}$ There are two main setup parts: the scanning unit and the signal-coupling unit. The scanning unit is responsible for providing the focused laser light at the correct radial position in the sample cell and collecting the signal emitted by the probe. At the same time it is able to perform a radial scan via a step motor, which is mounted on a linear translation stage. The signal-coupling unit focuses the collimated signal beam into a multimode glass fiber forwarding it to the spectrometer. A detailed list of components can be found in the ESI. $\dagger$ 

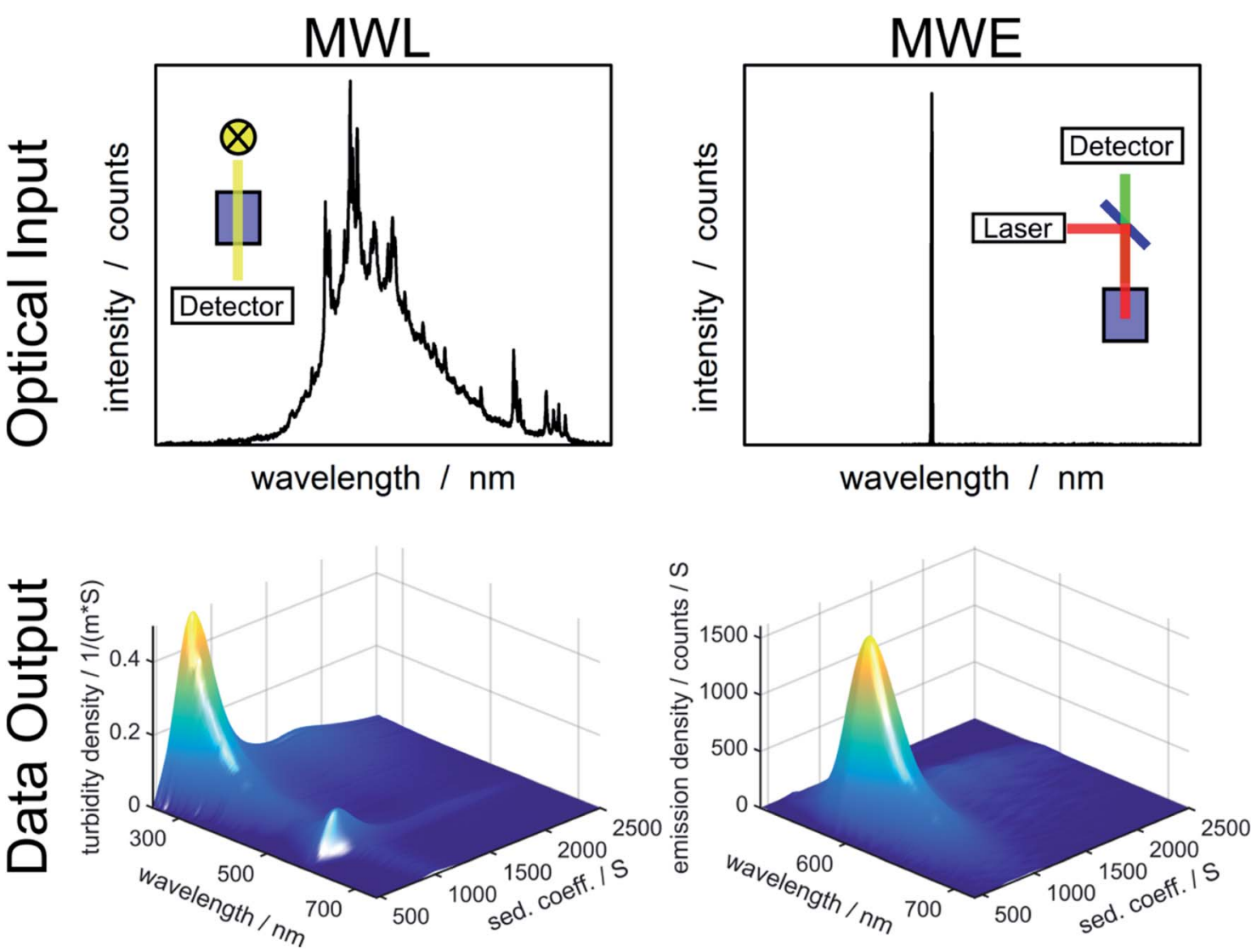

Fig. 1 Overview of optical input to the MWL- and MWE-AUC setups and data output from the analysis of an experiment with fluorescent labelled silica particles using HDR-MULTIFIT. ${ }^{4}$

The excitation light from the pump laser is coupled in a single-mode fiber and then fed into the vacuum chamber via a vacuum feedthrough. Using a laser diode allows direct amplitude modulation of the laser power via a TTL pulse from a timer card. Additionally, the laser pulse intensity can be adjusted by attenuating the amplitude of the TTL pulse via a simple potential divider based on a multi-turn potentiometer.
In the scanning unit (see Fig. 2), the laser light from the singlemode fiber is collimated with an off-axis parabolic (OAP) mirror M1 and is focused in the measurement cell via the OAP mirror M2 after passing through the central hole of OAP mirror M3. The light, emitted and backscattered (fluorescence, scattering and reflection) from the sample is collected and collimated by OAP mirror M3 and then focused by the OAP mirror M4 in a multi-

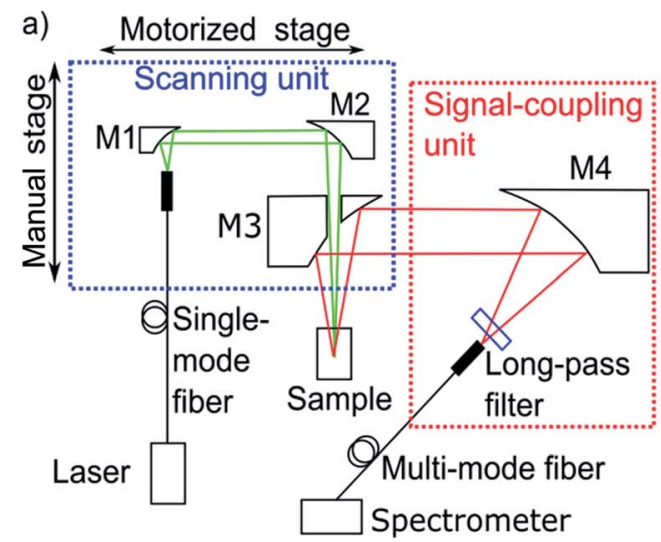

b)

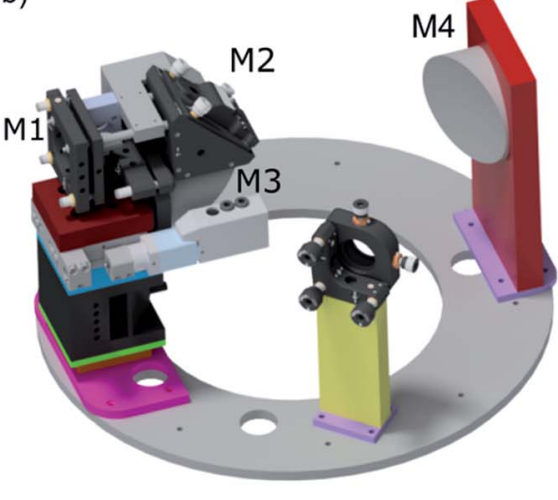

c)

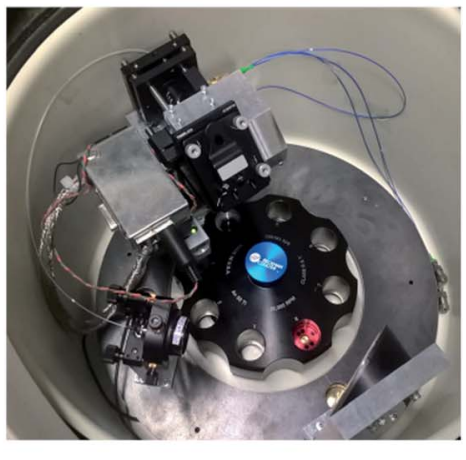

Fig. 2 (a) Main components of the optical setup projected in a single plane. (b) Three-dimensional depiction of setup (fibers are not shown). Optics and optomechanics are depicted with permission from Thorlabs ${ }^{32}$ and Newport, ${ }^{33}$ the linear actuator T-NA-SV2 is shown with permission from Zaber ${ }^{34}$ (http://www.zaber.com). (c) Photograph of the setup implemented in the vacuum chamber of the XL-80K centrifuge with fiber optics and electronics distributor box. 
mode step-index fiber after traveling through a spectral longpass filter. After the fiber vacuum feedthrough, the light is coupled into the spectrometer. Change of the scanning-unit position on the radial axis has no effect on light propagation because the beam is collimated. Furthermore, mirror M4 is larger than M3 to allow for different height adjustments of the scanning unit without changing the efficiency of light coupling into the multimode fiber. There are several advantages of using OPA mirrors instead of lenses for the fluorescence setup as used in the commercial fluorescence detector. ${ }^{28}$ The major benefit is the elimination of chromatic and spherical aberrations as it was already shown for the MWL-AUC. ${ }^{24}$ Additionally, the signal quality is improved due to the reduction of the fluorescence background. ${ }^{35}$ A drawback of the protected silver OAP mirrors is the decreasing reflectance below $90 \%$ for wavelengths lower than $400 \mathrm{~nm} .{ }^{32}$ With four OAP mirrors being used in the setup, the signal already decreases by $35 \%$. Changing to UV-enhanced aluminium OAP mirrors would allow covering also the UV range down to $250 \mathrm{~nm}$. However, in comparison to protected silver OAP mirrors, the reflectance in the visible range is reduced. The spectral experimental range is therefore currently limited by the optics to wavelengths above $400 \mathrm{~nm}$, while the upper wavelength limit is determined by the spectrometer at $1100 \mathrm{~nm}$. With different fibers, laser sources and spectrometer the range could be extended up to about $2500 \mathrm{~nm}$.

\section{Mechanical construction}

An Optima XL-80K ultracentrifuge from Beckman Coulter was modified to provide the possibility of fluorescence spectrum detection. Holes for the vacuum feedthroughs - for the glass fibers, for the electronics for the light barrier for angular position detection and for the step motor - were drilled in the heatsink. The scanning unit and the signal-coupling unit are both mounted on a laser-cut steel ring to facilitate accurate alignment of all components. The steel ring has an inner diameter of $192 \mathrm{~mm}$, a bit larger than the rotor, which means that it does not have to be placed below the rotor, thus avoiding any potential accident with an imbalanced rotor. The ring is mounted on three spacers, which themselves are screwed to the vacuum chamber. While mirror M4 is fixed in position and alignment, all other components are at least partly adjustable. The main part of the scanning unit is mounted on a cage system. The glass fiber delivering the laser light is directly mounted on a bracket, which is attached to a cage rod, shared by mirrors M1 and M2 and a lower cage rod, connected to mirror M1 only. Due to the restricted space and the holder of mirror M3, only the upper cage rods are used to connect the holders of mirrors M1 and M2. The connection of M3 with M1 and M2 is achieved by a custom aluminium part (see ESI $\dagger$ ) that also allows stabilizing M2 against tilting during the optical adjustment. To perform a radial scan, all the mirrors of the scanning unit - M1, M2, and M3 - are mounted on a linear translation stage with a step motor. This linear stage itself is mounted on a linear z-stage with manual translation, which enables to adjust the height of the scanning unit and therefore the focus position of the laser beam and, correspondingly, the detection volume in the sample. Mirror M4 is fixed on the steel ring, while the multi-mode fiber, which guides the detected light to the spectrometer, is attached to a 5-axis mount.

\section{Alignment procedure}

As the setup consists of two practically independent optical paths - the pump laser beam and that of emitted and backscattered light - at first each of them has to be adjusted individually. The mandatory superposition of both beams in the sample cell is to be provided at the end. The mirror M1 and the fiber tip in the FC/APC connector with tuneable focal position are adjusted to provide a circular collimated laser beam, which hits the middle of the mirror M2. The position of mirror M2 on the cage system is optimized to center the pump beam in the hole of mirror M3. Further adjustments are necessary to provide positioning of the excitation laser beam waist in the common focal plane of the mirrors M2 and M3 in the sample cell and to ensure the alignment of the beam straight in vertical direction.

An additional laser pointer, coupled to a single-mode fiber, can be used to invert the path of the scattered light for the adjustment of the 5-axis mount in the signal-coupling unit, as well as of the mirror M3 in the scanning unit for the best possible overlap of the laser beams over the common path in the sample cell. First, the 5-axis mount is adjusted in such a way that the laser beam hits the middle of mirror M4 by tilting the fiber tip. In a later step, the shape of the inverted laser spot at the sample position is made spherical by turning and tilting M3. Care should be taken that the optical axis of the inverted laser is also vertically aligned at the sample position. During alignment some turning of the mirrors might be necessary to provide a circular shape of the laser spots at the sample position. Finally, the two laser spots are brought together vertically and radially by fine adjusting the $x-y$ position of the fiber tip of the inverted laser as well as the tilting angle of mirror M2.

\section{Calibration}

AUC is a material-standard-free measurement technique. However, systematic errors may emerge from incorrect radial and angular positioning or temperature inaccuracy. ${ }^{36}$ Therefore, these parameters need to be carefully calibrated. Synchronization of spectrometer triggering and laser modulation with rotor rotation is achieved via a light barrier, triggering electronics and an adapted LabVIEW@-based data acquisition software developed previously. ${ }^{22}$ Details on the AUC triggering have been recently published elsewhere. ${ }^{31}$ In short, the temporal delay between the detection of a polished area on the rotor bottom via a light barrier and the sample cell arriving at the measurement position is a function of rotor speed and has to be calibrated using a fluorescent dye solution as a test sample.

Similarly, the radial position of the motorized stage needs to be evaluated. Therefore, radial calibration is performed with the counterbalance from Beckman Coulter. The counterbalance is installed in the rotor upside down and the reflection of the excitation laser light from the edges of the inner and outer mask is recorded at zero speed to avoid rotor stretching and loosening 
of the inverted weight. Turning the counterbalance is necessary to detect the reflection. More details on the radial and angular calibration can be found in the ESI. $\dagger$

Due to the several additional metal parts being located within the vacuum chamber, temperature control measurements at the top of the counterbalance at zero rotational speed were performed. ${ }^{37}$ The temperature control of the centrifuge shows a temperature offset of $+2{ }^{\circ} \mathrm{C}$, which is taken into account within the software, the experiment and the data evaluation. The deviation was already present prior to any modification of the centrifuge and can therefore be attributed to the centrifuge and not to the setup. Details on the temperature measurements can be found in the ESI. $\dagger$

\section{Validation of fluorescence signal}

Prior to any measurement, the spectral sensitivity of the new setup was evaluated. Therefore, the fluorescent dye rhodamine 6G was dissolved in water and its fluorescence spectra were measured under static and dynamic rotor conditions within the MWE-AUC and in the benchtop photoluminescence spectrometer. As one can see in Fig. 3, spectra obtained with the MWEAUC setup under static and dynamic conditions, are very similar and match also with the data obtained by the photoluminescence spectrometer. Small deviations in the red wing can be explained in terms of the spectral properties of optical components used, which exhibit some wavelength dependence of transmission and reflectance parameters.

Additionally, a concentration series of rhodamine $6 \mathrm{G}$ was used to assure the signal linearity of the acquired emission intensity. Measured extinction spectra enable to calculate the concentration of the fluorescent dye $\mathrm{e}^{38}$ in water and to correlate it with the maximum emission intensity as described in the ESI. $\uparrow$ Overall, a linear correlation for moderate concentrations ( $\approx \mu \mathrm{M}$ ) could be found (Fig. 3(a)), which allows correlating the emission intensity directly with the dye concentration.

\section{Confocal characteristics}

Radial and axial resolutions are important for signal generation and data evaluation. The meniscus ("liquid edge") of a fluorescent dye solution in water can be used to estimate the radial resolution of the setup. ${ }^{24}$ In comparison to the cell bottom or the counterbalance, it gives larger values for the radial resolution for MWL-AUC. ${ }^{24}$ The radial intensity distributions for the excitation and a few emission wavelengths are given in Fig. 3(b). Notably, the intensity of the laser light $(518 \mathrm{~nm})$ reflected from the bottom surface of the upper cell window, which changes due to the change in refractive index at the gas-water-glass interface, gives a similar indication of the meniscus position as the fluorescence signal. Additionally, the fluorescence intensity distribution is similar for different wavelengths as a consequence of the used chromatic aberration-free parabolic mirrors. The distribution width between $10 \%$ and $90 \%$ intensity is approximately $100 \mu \mathrm{m}$.

The same sample can also be used to characterize the axial confocal parameter in static mode. Therefore, the height of the scanning unit was adjusted via the linear z-stage and the mean emission intensity (522-700 nm) was recorded. From the results in Fig. 3, the axial resolution can be approximated to be $\approx 2$ $\mathrm{mm}$.

\section{Sedimentation velocity experiment}

A protein with attached fluorescence dye was used to characterize the performance of the MWE-AUC setup. The acquired data was analyzed with SEDFIT using the built-in fluorescence tools $^{\mathbf{1 8}}$ (see Fig. 4). The measured data is very similar to the already reported datasets of fluorescence without spectral discrimination, ${ }^{\mathbf{1 8}}$ including an increase of signal magnitude with radius. This effect is considered to originate from a shift of the focal point during the radial movement of the detector setup $^{18,39}$ but can be well accounted for in SEDFIT. The major improvement in comparison to the established fluorescence
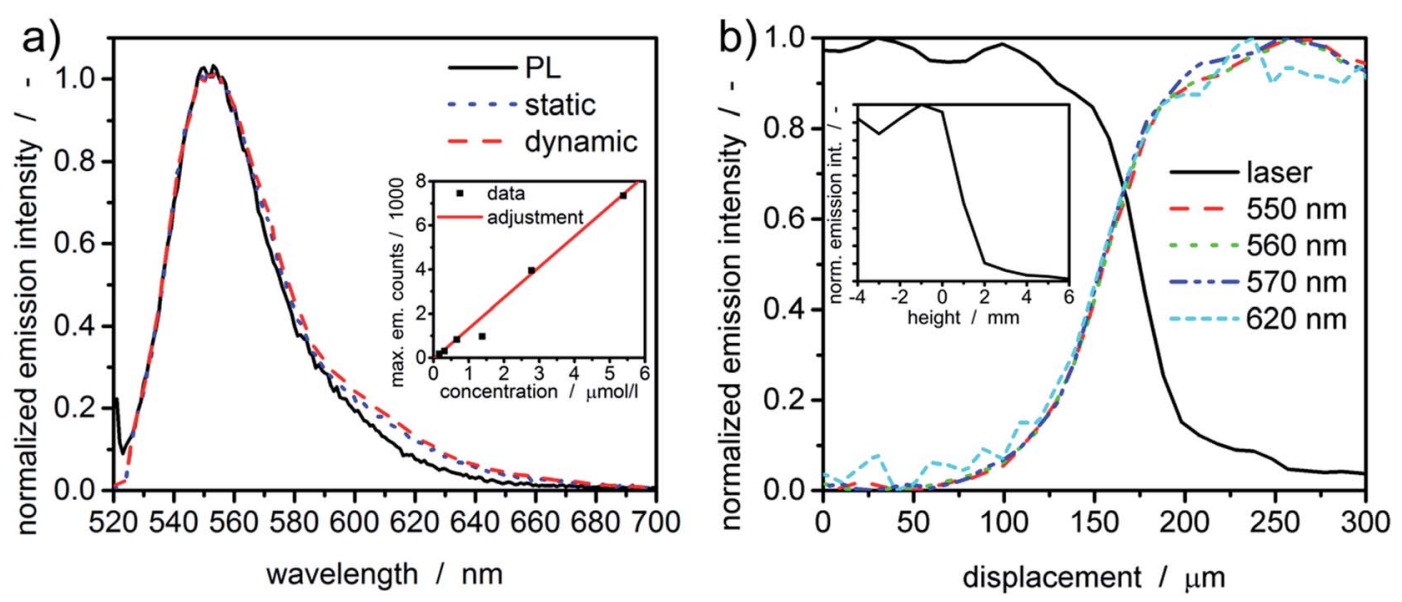

Fig. 3 (a) Normalized emission spectra of rhodamine 6G acquired with MWE-AUC under static conditions and within a constant radius experiment (dynamic) as well as obtained with the benchtop photoluminescence spectrometer ( $\mathrm{PL}$ ). The inset shows the linear correlation between fluorescence signal and the concentration of fluorescent dye. The data point at $\approx 1.4 \mu \mathrm{mol} \mathrm{L} \mathrm{L}^{-1}$ was excluded from the adjustment. (b) Normalized intensity signal for a single radial scan of a liquid edge of dissolved rhodamine $6 \mathrm{G}$ at $10000 \mathrm{rpm}$. The inset shows the emission signal as a function of the focus height position within the sample under static conditions. 
a)

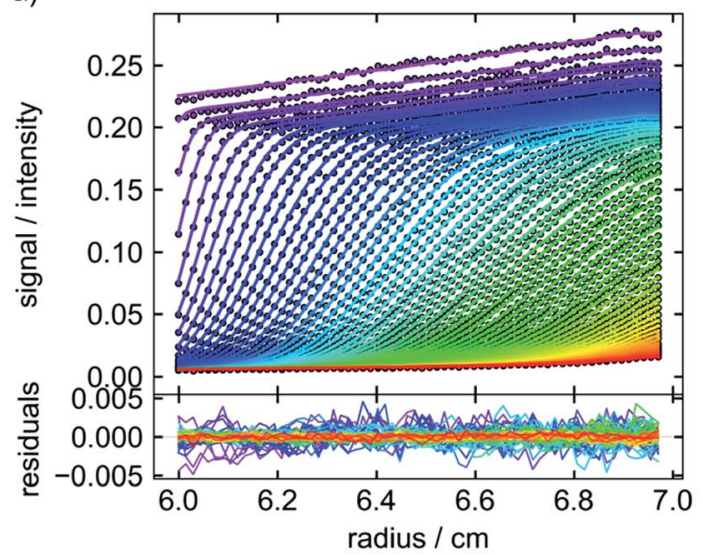

b)

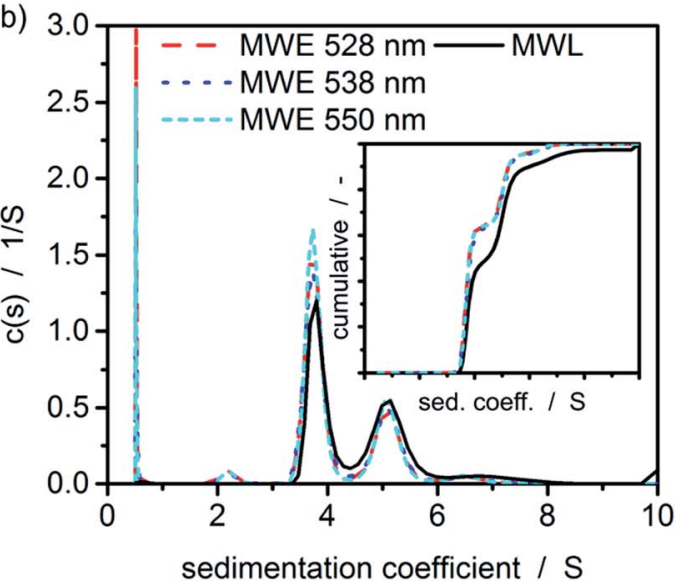

Fig. 4 (a) Raw data of the MWE-AUC experiment of the albumin-fluorescein isothiocyanate conjugate at $550 \mathrm{~nm}$ and a rotor speed of $40000 \mathrm{rpm}$ with model curves from the c(s) method implemented in SEDFIT. ${ }^{18,41}$ (b) Comparison of c(s) distribution obtained via MWL-AUC at $495 \mathrm{~nm}\left(f / f_{0}=1.67\right)$ and MWE-AUC $\left(f / f_{0}=1.66,1.66\right.$, and 1.64) for different wavelengths from the analysis in SEDFIT. The inset shows the cumulative sedimentation coefficient distributions neglecting species below $3 \mathrm{~S}$.

AUC is the now available spectral data. The resulting sedimentation coefficient distributions in Fig. 4 and the fitted frictional ratios $\left(f l f_{0}=1.66,1.66\right.$, and 1.64 for $528 \mathrm{~nm}, 538 \mathrm{~nm}$ and $550 \mathrm{~nm}$, accordingly) are very similar for different evaluation wavelengths. This means that as expected for uniform samples, sedimentation and diffusion properties can be analyzed equally well for different wavelengths of the fluorescence spectrum. This is the prerequisite for the simultaneous hydrodynamic, thermodynamic and spectral characterization of macromolecules and nanoparticles within the MWE-AUC. A depiction of the corresponding raw data and an exemplary spectrum can be found in the ESI. $\dagger$ In order to fully validate the setup and due to the fact that the fluorescent dye might change the hydrodynamic and thermodynamic properties of the protein, the sample was also studied with the MWL-AUC setup. The comparison of the results obtained from MWE-AUC for different wavelengths and the MWL-AUC setup is depicted in Fig. 4 . The position of the three main species and the fitted frictional ratio $\left(f \mid f_{0}=1.67\right.$ for MWL-AUC) can be well retrieved, meaning that sedimentation and diffusion coefficients are in excellent agreement between MWE-AUC and MWL-AUC. Slight differences occur for species below 3S. Most likely, these correspond to the fluorescent dye that desorbed from the protein, either at the preparation stage or during the experiment. As the same sample was investigated, both setups should deliver similar results for the relative concentration of the free dye. However, in the MWL-AUC, the relative dye concentration amounts to less than $1 \%$, while the MWE-AUC gives values up to $8 \%$. It is well known that results from different AUC detection systems may indeed be slightly altered. ${ }^{36}$ There are several reasons to explain that. First of all, the different detection systems exhibit different concentration ranges that appear convenient for thorough analysis. Secondly, distributions generally depend on the measurement technique used, as fractions of particles may contribute differently to the overall acquired signal for different detectors. ${ }^{40}$ Thus, due to the different emission properties of covalently bound and free fluorophores, the relative signal intensities can be altered. Another difference between MWE- and MWL-AUC is photodegradation that is present only within the MWE-AUC. Due to the small size of the free fluorescent dye, it does not show significant sedimentation at the applied rotor speed but is constantly subjected to excitation. Therefore, a decrease of emission due to photodegradation could be misinterpreted as sedimenting species.

\section{Constant radius experiment}

In order to test the performance of the setup in terms of spectral sensitivity and higher values of sedimentation coefficients, fluorescent silica particles were characterized using a constant radius experiment., ${ }^{4,15}$ The constant radius experiment is chosen as it allows an easier access to the spectral properties of a sample. Moreover, the radial characteristics of the fluorescent setup, as shown in Fig. 4, do not play a significant role due to the static measurement position. Fluorescent silica particles were measured both with MWL-AUC and MWE-AUC. In order to avoid any deviation of sedimentation coefficient distributions due to the size-dependent scattering of the silica particles, the evaluation of the sedimentation data from MWL-AUC was undertaken at the extinction peak of the fluorescent dye. However, due to the boundary conditions of the two setups, different concentrations $\left(1 \mathrm{mg} \mathrm{mL}^{-1}\right.$ in MWE- and $5 \mathrm{mg} \mathrm{mL}^{-1}$ in MWL-AUC) had to be investigated. Otherwise, the optical signal of the MWE-AUC would be compromised by the inner filter effect or the MWL-AUC would not be able to measure sufficiently high extinction signals. The free fluorescent dye, which does not show meniscus depletion, can be used after the measurement to detect the radial position of the meniscus, which is necessary for data evaluation.

Results for the sedimentation coefficient as well as the spectra extracted from HDR-MULTIFIT ${ }^{4}$ can be found in Fig. 5 . The excellent agreement of the sedimentation coefficient distributions of MWL-AUC and MWE-AUC shows that the new device is working properly and provides accurate results not 

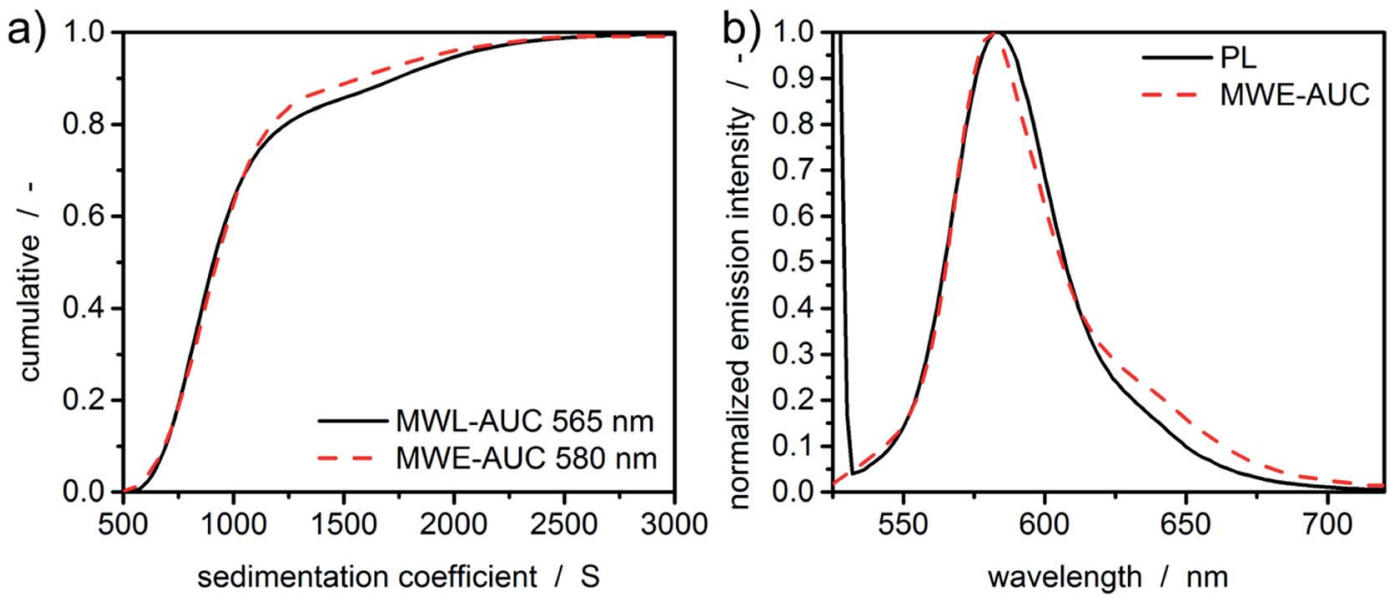

Fig. 5 (a) Sedimentation coefficient distributions for the fluorescent silica particles obtained by MWL-AUC and MWE-AUC via HDR-MULTIFIT. (b) Emission spectra extracted from the constant radius experiment for the entire sedimentation coefficient distribution compared to the results from the benchtop photoluminescence spectrometer (PL).

only in terms of sedimentation coefficients but also in terms of the detected concentration ratios. Additionally, it can be seen that the spectra extracted from the sedimentation coefficient distributions are matching the spectra obtained by the benchtop photoluminescence spectrometer, showing only a minor deviation on the red spectrum side, similar to Fig. 3.

\section{Fluorescence emission of graphene oxide and quantum dots}

So far, the acquired fluorescence spectra originated from the fluorescent dyes. Particularly interesting is the analysis of particulate samples that show intrinsic fluorescence. To illustrate this, an ultrasound-processed sample of graphene oxide was investigated in a constant radius experiment. Graphene as a zero-bandgap semiconductive material does not display any photoluminescence. However, introducing functional groups such as hydroxy groups or epoxides opens the bandgap, giving new routes for determining its structure using fluorescence measurements.

With the graphene oxide being a monolayer material, the sedimentation velocity is directly related to the lateral dimension of the platelet. Therefore, sedimentation coefficients can be directly transformed to equivalent diameters using the method and parameters published in literature. ${ }^{6}$ As can be seen in Fig. 6(a), even broad size distributions with sizes up to the micrometer range can be measured in accordance with typical samples originating from this synthetic route. ${ }^{42}$ The fluorescence spectrum of graphene oxide shows typical green emission in the range of $500-700 \mathrm{~nm}^{43,44}$ Graphene oxide is a network of non-uniform regions with $\mathrm{sp}^{2}$ - and $\mathrm{sp}^{3}$-hybridized carbon atoms. In such materials, a mixture of $\mathrm{sp}^{2}$ - and $\mathrm{sp}^{3}$-bondings determines the opto-electronic properties by the $\pi$ states of the $\mathrm{sp}^{2}$-sites. The green to infrared emission region $(500-800 \mathrm{~nm})$ as shown in Fig. 6(b) is suggested to originate from localized
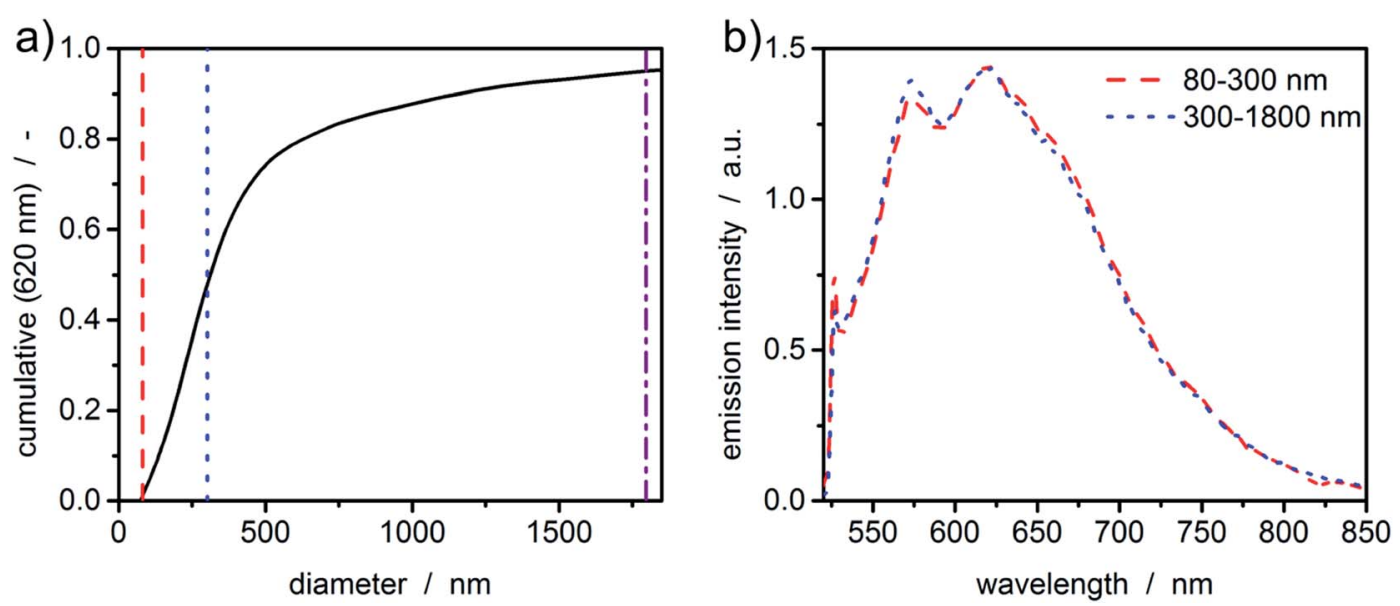

Fig. 6 Results of the constant radius experiment for the processed graphene oxide sample obtained via HDR-MULTIFIT. (a) Cumulative diameter distribution obtained from the sedimentation coefficient distribution at $620 \mathrm{~nm}$. (b) Extracted spectra of the graphene oxide platelets. Colours and dashed and dotted lines are corresponding to the lower boundaries of the respective size interval in (a). 

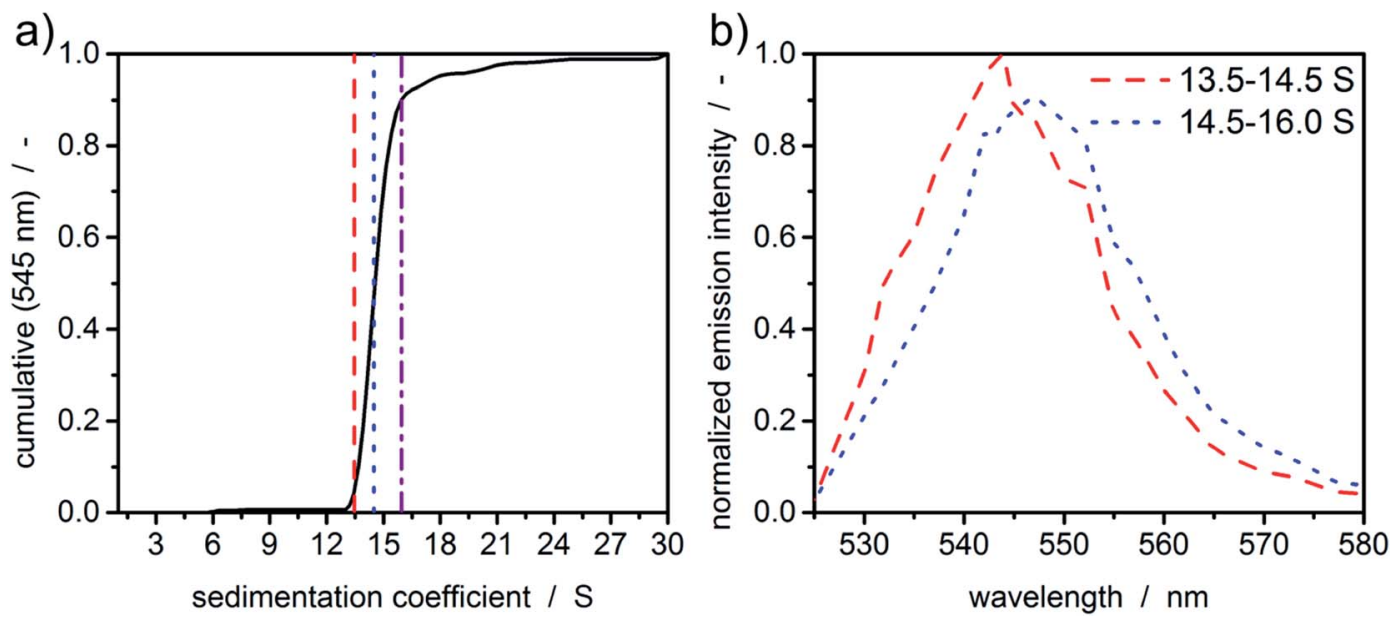

Fig. 7 (a) Cumulative c(s) distribution of CdSe/ZnS quantum dots at $545 \mathrm{~nm}$ and sedimentation coefficient intervals used for the extraction of fluorescence spectra. (b) Extracted normalized spectra of the quantum dots. Colour and dashed and dotted lines are similar to the lower boundary of the respective interval in (a).

electronic structure, neighbouring functional groups or can refer to $\mathrm{sp}^{2}$ carbon regions captured by repulsive $\mathrm{sp}^{3}$ carbon hard wall barriers. Further, the bond alteration inducing intervalley scattering effects can also cause the emission. ${ }^{45}$ The intensity of the photoluminescence correlates with the degree of defects in the carbon framework.

Although the sample is polydisperse, hardly any deviations of the fluorescence signal for the two intervals can be observed. This leads to the conclusion that the sample is quite uniform in terms of the chemical composition.

In order to demonstrate the potential of MWE-AUC with respect to the analysis of size- and shape-dependent emission properties of nanoparticles, we show in Fig. 7 exemplary results for CdSe/ZnS quantum dots, where the emission intensity as well as the spectral peak position depends on the CdSe core size as well as on the thickness of the $\mathrm{ZnS}$ shell. ${ }^{46}$ As shown in Fig. 7(a), the cumulative sedimentation coefficient distribution is very narrow, therefore only two intervals with equal increment between 0.05 and 0.9 of the cumulative distribution are used for the extraction of spectra. The values for the interval boundaries were chosen to exclude small side products and agglomerates based on the shape of the sedimentation coefficient distribution. Fig. 7(b) gives a clear indication of a sedimentation coefficient dependent spectral shift of only $3 \mathrm{~nm}$, which demonstrates the ability of the MWE-AUC setup. The red shift can be explained with larger core sizes and/or shell thickness, which is plausible to be correlated with higher sedimentation coefficients. On this basis, complex structureproperty relationships can be studied now in unprecedented detail via MWE-AUC.

\section{Conclusion}

A new multiwavelength emission detector for analytical ultracentrifugation (MWE-AUC) was developed and is presented in this paper. The analysis of fluorescent-labelled proteins and silica particles, graphene oxide and CdSe/ZnS quantum dots demonstrated the capability of hydrodynamic and optical characterization of nanoparticles and macromolecules using the new MWE-AUC setup. Hence, new and so far inaccessible applications could be the stoichiometric study of multi-protein complexes and their binding behaviour, the identification of different species in carbon allotropes such as carbon nanodots or graphene quantum dots. In particular, the fluorescence spectra can provide details on size- and structure-dependent emission characteristics of quantum materials as it has been shown for the exemplary case of core-shell CdSe/ZnS quantum dots. Now a comprehensive toolbox for the optical characterization of nanoparticles by combined analysis of size- and shape-dependent extinction (MWL-AUC) and fluorescence (MWE-AUC) properties is available.

\section{Conflicts of interest}

There are no conflicts to declare.

\section{Acknowledgements}

The authors acknowledge the funding of the Deutsche Forschungsgemeinschaft (DFG) through project PE 427/37-1 and the Collaborative Research Center SFB 953: "Synthetic Carbon Allotropes" and thank Dr Wendel Wohlleben and Michael Kaiser from BASF SE for providing custom-made AUC accessories. M. M. and S. E. thank DFG for funding through Project 392444269.

\section{References}

1 M. L. Mastronardi, F. Maier-Flaig, D. Faulkner, E. J. Henderson, C. Kübel, U. Lemmer and G. A. Ozin, Sizedependent absolute quantum yields for size-separated colloidally-stable silicon nanocrystals, Nano Lett., 2012, 12, 337-342. 
2 I. Moreels, K. Lambert, D. de Muynck, F. Vanhaecke, D. Poelman, J. C. Martins, G. Allan and Z. Hens, Composition and Size-Dependent Extinction Coefficient of Colloidal PbSe Quantum Dots, Chem. Mater., 2007, 19, 6101-6106.

3 S. E. Wawra, L. Pflug, T. Thajudeen, C. Kryschi, M. Stingl and W. Peukert, Determination of the two-dimensional distributions of gold nanorods by multiwavelength analytical ultracentrifugation, Nat. Commun., 2018, 9, 4898.

$4 \mathrm{~J}$. Walter and W. Peukert, Dynamic range multiwavelength particle characterization using analytical ultracentrifugation, Nanoscale, 2016, 8, 7484-7495.

5 J. Zhang, J. Z. Pearson, G. E. Gorbet, H. Cölfen, M. W. Germann, M. A. Brinton and B. Demeler, Spectral and Hydrodynamic Analysis of West Nile Virus RNAProtein Interactions by Multiwavelength Sedimentation Velocity in the Analytical Ultracentrifuge, Anal. Chem., 2017, 89, 862-870.

6 J. Walter, T. J. Nacken, C. Damm, T. Thajudeen, S. Eigler and W. Peukert, Determination of the lateral dimension of graphene oxide nanosheets using analytical ultracentrifugation, Small, 2015, 11, 814-825.

7 T. Svedberg and H. Rinde, The Ultra-Centrifuge, A New Instrument For The Determination Of Size And Distribution Of Size Of Particle In Amicroscopic Colloids, J. Am. Chem. Soc., 1924, 46(12), 2677-2693.

8 J. W. Beams, N. Snidow, A. Robeson and H. M. Dixon, Interferometer for the Measurement of Sedimentation in a Centrifuge, Rev. Sci. Instrum., 1954, 25, 295-296.

9 W. Mächtle and U. Klodwig, A New 8-Cell-Interference and Schlieren Optics Multiplexer for the Analytical Ultracentrifuge Based upon a Modulable Laser and a High Speed 8-Hole-Rotor with $60000 \mathrm{rpm}$, Makromol. Chem., 1979, 180, 2507-2511.

10 R. H. Crepeau, R. H. Conrad and S. J. Edelstein, UV laser scanning and fluorescence monitoring of analytical ultracentrifugation with an on-line computer system, Biophys. Chem., 1976, 5, 27-39.

11 B. Schmidt, W. Rappold, V. Rosenbaum, R. Fischer and D. Riesner, A fluorescence detection system for the analytical ultracentrifuge and its application to proteins, nucleic acids, and viruses, Colloid Polym. Sci., 1990, 268, 45-54.

12 I. K. MacGregor, A. L. Anderson and T. M. Laue, Fluorescence detection for the XLI analytical ultracentrifuge, Biophys. Chem., 2004, 108, 165-185.

13 A. Balbo, K. H. Minor, C. A. Velikovsky, R. A. Mariuzza, C. B. Peterson and P. Schuck, Studying multiprotein complexes by multisignal sedimentation velocity analytical ultracentrifugation, Proc. Natl. Acad. Sci. U. S. A., 2005, 102, $81-86$.

14 S. K. Bhattacharyya, P. Maciejewska, L. Börger, M. Stadler, A. M. Gülsün, H. B. Cicek and H. Cölfen, in Analytical Ultracentrifugation VIII, ed. C. Wandrey and H. Cölfen, Springer-Verlag GmbH, Berlin Heidelberg, 2006, pp. 9-22.

$15 \mathrm{~W}$. Scholtan and H. Lange, Bestimmung der Teilchengrößenverteilung von Latices mit der
Ultrazentrifuge, Kolloid-Zeitschrift und Zeitschrift für Polymere, 1972, 250, 782-796.

16 W. Mächtle, High-resolution, submicron particle size distribution analysis using gravitational-sweep sedimentation, Biophys. J., 1999, 76, 1080-1091.

17 H. Zhao, Y. Fu, C. Glasser, E. J. A. Alba, M. L. Mayer, G. Patterson and P. Schuck, Monochromatic multicomponent fluorescence sedimentation velocity for the study of high-affinity protein interactions, eLife, 2016, 5, e17812.

18 H. Zhao, E. Casillas, H. Shroff, G. H. Patterson and P. Schuck, Tools for the quantitative analysis of sedimentation boundaries detected by fluorescence optical analytical ultracentrifugation, PLoS One, 2013, 8, e77245.

19 W. Schrof, P. Rossmanith and W. Mächtle, Centrifuging process for sample characterization, US6278518B1, 2001.

20 S. Eigler, M. Enzelberger-Heim, S. Grimm, P. Hofmann, W. Kroener, A. Geworski, C. Dotzer, M. Röckert, J. Xiao, C. Papp, O. Lytken, H.-P. Steinrück, P. Müller and A. Hirsch, Wet chemical synthesis of graphene, $A d v$. Mater., 2013, 25, 3583-3587.

21 B. Butz, C. Dolle, C. E. Halbig, E. Spiecker and S. Eigler, Highly Intact and Pure Oxo-Functionalized Graphene: Synthesis and Electron-Beam-Induced Reduction, Angew. Chem., 2016, 55, 15771-15774.

22 J. Walter, K. Löhr, E. Karabudak, W. Reis, J. Mikhael, W. Peukert, W. Wohlleben and H. Cölfen, Multidimensional analysis of nanoparticles with highly disperse properties using multiwavelength analytical ultracentrifugation, ACS Nano, 2014, 8, 8871-8886.

23 P. Schuck, Size-Distribution Analysis of Macromolecules by Sedimentation Velocity Ultracentrifugation and Lamm Equation Modeling, Biophys. J., 2000, 78, 1606-1619.

24 J. Pearson, J. Walter, W. Peukert and H. Cölfen, Advanced Multiwavelength Detection in Analytical Ultracentrifugation, Anal. Chem., 2018, 90, 1280-1291.

25 P. Schuck and P. Rossmanith, Determination of the sedimentation coefficient distribution by least-squares boundary modeling, Biopolymers, 2000, 54, 328-341.

26 W. Mächtle and L. Börger, Analytical Ultracentrifugation of Polymers and Nanoparticles, Springer-Verlag, s.l., 1st edn, 2006.

27 P. W. Schuck, H. Zhao, C. A. Brautigam and R. Ghirlando, Basic Principles of Analytical Ultracentrifugation, CRC Press Taylor \& Francis Group, Boca Raton, London, New York, 2015.

28 Analytical Ultracentrifugation. Instrumentation, Software, and Applications, ed. S. Uchiyama, F. Arisaka, W.F. Stafford and T. Laue, Springer Japan, Tokyo, s.l., 2016.

29 O. Lamm, Die differentialgleichung der ultrazentrifugierung, Almqvist \& Wiksell, 1929.

30 T. Svedberg, Zentrifugierung, Diffusion und Sedimentationsgleichgewicht von Kolloiden und hochmolekularen Stoffen, Kolloid-Z., 1925, 36, 53-64.

$31 \mathrm{~J}$. Pearson and $\mathrm{H}$. Cölfen, Practical Aspects of Multiwavelength Analytical Ultracentrifugation, Instruments, 2019, 3, 23. 
32 Thorlabs GmbH, Thorlabs. Available at https://www.thorlabs.de/.

33 Newport Corporation, Newport. Available at https:// www.newport.com/.

34 Zaber Technologies Inc, Zaber. Available at https:// www.zaber.com/.

35 S. Rupp, A. Off, H. Seitz-Moskaliuk, T. M. James and H. H. Telle, Improving the Detection Limit in a Capillary Raman System for In Situ Gas Analysis by Means of Fluorescence Reduction, Sensors, 2015, 15, 23110-23125.

36 H. Zhao, R. Ghirlando, C. Alfonso, F. Arisaka, I. Attali, D. L. Bain, M. M. Bakhtina, D. F. Becker, G. J. Bedwell, A. Bekdemir, T. M. D. Besong, C. Birck, C. A. Brautigam, W. Brennerman, O. Byron, A. Bzowska, J. B. Chaires, C. T. Chaton, H. Cölfen, K. D. Connaghan, K. A. Crowley, U. Curth, T. Daviter, W. L. Dean, A. I. Díez, C. Ebel, D. M. Eckert, L. E. Eisele, E. Eisenstein, P. England, C. Escalante, J. A. Fagan, R. Fairman, R. M. Finn, W. Fischle, J. G. de La Torre, J. Gor, H. Gustafsson, D. Hall, S. E. Harding, J. G. H. Cifre, A. B. Herr, E. E. Howell, R. S. Isaac, S.-C. Jao, D. Jose, S.-J. Kim, B. Kokona, J. A. Kornblatt, D. Kosek, E. Krayukhina, D. Krzizike, E. A. Kusznir, H. Kwon, A. Larson, T. M. Laue, A. Le Roy, A. P. Leech, H. Lilie, K. Luger, J. R. LuqueOrtega, J. Ma, C. A. May, E. L. Maynard, A. Modrak-Wojcik, Y.-F. Mok, N. Mücke, L. Nagel-Steger, G. J. Narlikar, M. Noda, A. Nourse, T. Obsil, C. K. Park, J.-K. Park, P. D. Pawelek, E. E. Perdue, S. J. Perkins, M. A. Perugini, C. L. Peterson, M. G. Peverelli, G. Piszczek, G. Prag, P. E. Prevelige, B. D. E. Raynal, L. Rezabkova, K. Richter, A. E. Ringel, R. Rosenberg, A. J. Rowe, A. C. Rufer, D. J. Scott, J. G. Seravalli, A. S. Solovyova, R. Song, D. Staunton, C. Stoddard, K. Stott, H. M. Strauss, W. W. Streicher, J. P. Sumida, S. G. Swygert, R. H. Szczepanowski, I. Tessmer, R. T. Toth, A. Tripathy, S. Uchiyama, S. F. W. Uebel, S. Unzai, A. V. Gruber, P. H. von Hippel, C. Wandrey, S.-H. Wang, S. E. Weitzel, B. Wielgus-Kutrowska, C. Wolberger, M. Wolff, E. Wright, Y.-S. Wu, J. M. Wubben and P. Schuck, A multilaboratory comparison of calibration accuracy and the performance of external references in analytical ultracentrifugation, PLoS One, 2015, 10, e0126420.

37 H. Zhao, A. Balbo, H. Metger, R. Clary, R. Ghirlando and P. Schuck, Improved measurement of the rotor temperature in analytical ultracentrifugation, Anal. Biochem., 2014, 451, 69-75.

38 S. Terdale and A. Tantray, Spectroscopic study of the dimerization of rhodamine $6 \mathrm{G}$ in water and different organic solvents, J. Mol. Liq., 2017, 225, 662-671.

39 R. R. Kroe and T. M. Laue, NUTS and BOLTS: applications of fluorescence-detected sedimentation, Anal. Biochem., 2009, 390, 1-13.

40 U. Frank, S. E. Wawra, L. Pflug and W. Peukert, Multidimensional Particle Size Distributions and Their Application to Nonspherical Particle Systems in Two Dimensions, Part. Part. Syst. Charact., 2019, 1800554.

41 C. A. Brautigam, in Methods in enzymology, Elsevier, 2015, pp. 109-133.

42 T. J. Nacken, C. E. Halbig, S. E. Wawra, C. Damm, S. Romeis, J. Walter, M. J. Tehrani, Y. Hu, Y. Ishii, S. Eigler and W. Peukert, Structural factors controlling size reduction of graphene oxide in liquid processing, Carbon, 2017, 125, 360-369.

43 G. Eda, Y.-Y. Lin, C. Mattevi, H. Yamaguchi, H.-A. Chen, I.-S. Chen, C.-W. Chen and M. Chhowalla, Blue photoluminescence from chemically derived graphene oxide, Adv. Mater., 2010, 22, 505-509.

44 V. Strauss, R. A. Schäfer, F. Hauke, A. Hirsch and D. M. Guldi, Polyhydrogenated Graphene: Excited State Dynamics in Photo- and Electroactive Two-Dimensional Domains, J. Am. Chem. Soc., 2015, 137, 13079-13086.

45 Z. Luo, P. M. Vora, E. J. Mele, A. T. C. Johnson and J. M. Kikkawa, Photoluminescence and band gap modulation in graphene oxide, Appl. Phys. Lett., 2009, 94, 111909.

46 B. O. Dabbousi, J. Rodriguez-Viejo, F. V. Mikulec, J. R. Heine, H. Mattoussi, R. Ober, K. F. Jensen and M. G. Bawendi, (CdSe)ZnS Core-Shell Quantum Dots: Synthesis and Characterization of a Size Series of Highly Luminescent Nanocrystallites, J. Phys. Chem. B, 1997, 101, 9463-9475. 